\title{
Balancing Student Sex Education Needs with Community Preferences: A Qualitative Study in Rural Indiana
}

Sarah Hoseus ${ }^{1}$, Carolyn G. Meagher ${ }^{2}$, Rebecca James ${ }^{3}$, Doug Cope-Barnes ${ }^{3}$, Mary A. Ott ${ }^{2}$ ${ }^{1}$ Indiana University School of Medicine; ${ }^{2}$ Indiana University School of Medicine, Department of Pediatrics, Division of Adolescent Medicine; ${ }^{3}$ Health Care Education and Training, Inc.

Background and Purpose: Rural youth experience high rates of teen pregnancy. Despite this increased health risk, little data exist on evidence-based sex education programs (EBPs) in rural communities. Using the Exploration, Preparation, Implementation, and Sustainment (EPIS) framework, we describe the challenges and community-derived solutions in implementing an EBP in rural Indiana schools, providing insight on rural EBP implementation.

Methods: As part of a larger federally funded implementation project, an abstinence focused EBP (Making a Difference! and Guiding Good Choices) was taught at middle schools located in twelve counties in rural southern and eastern Indiana. Three community agencies implemented the curriculum over three years. Afterwards, $\mathrm{SH}$ and $\mathrm{CM}$ conducted interviews of the agency partners $(n=13)$ and of key school stakeholders $(n=6)$. Interviews were conducted via Zoom, audio-recorded, transcribed, field notes written, and all data analyzed using thematic analysis. Codes were developed from the EPIS framework and included outer contexts of communities, inner contexts of schools, preparation activities, implementation, and potential sustainability. We asked participants to identify challenges and solutions.

Results: Challenges and solutions were mapped onto the EPIS framework. Outer context challenges included religion, conservative communities, outside agencies, and parenting beliefs. Inner context challenges included lack of teacher and administrator support. Preparational challenges included lack of communication with parents, lack of community outreach, and lack of parent education. Implementational challenges included teaching a 60-minute lesson in 45 minutes and classroom engagement of schoolteachers. Sustainment challenges included teacher discomfort with the topic. Community-derived solutions included building community trust before implementation, providing information to parents, inviting administrators to observe, offering a student question box, and training school staff to implement the program.

Conclusion and Potential Impact: Many challenges emerged during implementation because of perceived lack of preparation. Future implementation of EBPs in rural communities should focus more on aspects of exploration and preparation. 